\title{
Nonlocality without inequalities for almost all entangled states of any quantum system.*
}

\author{
GianCarlo Ghirardi ${ }^{1,2,3, \text {, }}$ and Luca Marinatto ${ }^{1,2}$, 目 \\ ${ }^{1}$ Department of Theoretical Physics of the University of Trieste, Italy \\ ${ }^{2}$ Istituto Nazionale di Fisica Nucleare, Sezione di Trieste, Italy \\ ${ }^{3}$ International Centre for Theoretical Physics "Abdus Salam," Trieste, Italy
}

(Dated: November 23, 2018)

\begin{abstract}
It is shown that it is possible to rule out all local and stochastic hidden variable models accounting for the quantum mechanical predictions implied by almost any entangled quantum state vector of any number of particles whose Hilbert spaces have arbitrary dimensions, without resorting to Belltype inequalities. The present proof makes use of the mathematically precise notion of Bell locality and it involves only simple set theoretic arguments.

PACS numbers: 03.65.Ud
\end{abstract}

In a seminal paper 1], L. Hardy has shown that for all entangled, but not maximally entangled, states of two spin-1/2 particles, one can prove nonlocality without resorting to inequalities. More precisely he proved that "local realism" unavoidably conflicts with the quantum predictions by resorting to typical EPR-counterfactual arguments [2]. Hardy's paper can be considered the best example of the attempts of proving nonlocality without using inequalities and, as such, it has been defined "the best version of Bell's theorem" [3]. Such an approach was initiated by the celebrated GHZ argument [4] with reference to a precise entangled state of three spin- $1 / 2$ particles. Subsequently S. Goldstein [5] simplified the proof of Ref. 1] and extended it to the case of bipartite systems whose constituents belong to Hilbert spaces of arbitrary dimensions. Later on, new refinements $[\underline{6}$ ) of the original argument by Hardy, trying to maximize the probability of certain quantum mechanical outcomes which cannot be accounted for by any local deterministic hidden variable model, have been presented.

The aim of this paper is to reformulate and generalize Hardy and Goldstein's arguments by exhibiting, without resorting to inequalities, a contradiction between the existence of a local stochastic hidden variable model and the quantum mechanical predictions for almost any non-completely factorizable state vector of a system composed of an arbitrary number of particles whose Hilbert spaces have arbitrary dimensionality. Our formal approach, inspired by the works of Refs. [1, 5], is particularly simple and straightforward since it involves only elementary set theoretic arguments avoiding the use of counterfactuals, and it is completely general. For what concerns the experimental implications, our approach has the advantage of identifying the precise set of measurements which can yield, with a precise probability, outcomes whose occurrence put into evidence the unavoidable "non-locality" of every conceivable hidden variable completion of quantum mechanics. Contrary to all the nonlocality tests based on a violation of some Bell's inequality which require different correlation experiments, the tests based on a Hardy-like argument require simply the occurrence of a unique and particular joint event, which is unambiguously identified once one knows the state he is supplied with.

Let us start by making precise the formal framework we are dealing with and the locality request we will use. The framework will be the one of the so-called stochastic hidden variable theories for correlation experiments of the EPR-Bell type which involve measurement processes performed on a $n$-partite quantum system in the state $|\psi(1,2, \ldots, n)\rangle \in \mathcal{H}_{1} \otimes \cdots \otimes \mathcal{H}_{n}$ (where the dimensionality of $\mathcal{H}_{i}$ is arbitrary). It consists of: (i) a set $\Lambda$ whose elements $\lambda$ are called hidden variables; (ii) a normalized and positive probability distribution $\rho$ defined on $\Lambda$; (iii) a set of probability distributions $P_{\lambda}\left(A_{i}=a, B_{j}=b, \ldots, Z_{k}=z\right)$ for the outcomes of single and joint measurements of any conceivable set of observables $\left\{A_{i}, B_{j}, \ldots, Z_{k}\right\}$ where each index $\{i, j, \ldots, k\}$ refers to a single particle or to a group of particles, such that:

$$
P_{\psi}\left(A_{i}=a, B_{j}=b, \ldots, Z_{k}=z\right)=\int_{\Lambda} d \lambda \rho(\lambda) P_{\lambda}\left(A_{i}=a, B_{j}=b, \ldots, Z_{k}=z\right) .
$$

Here the quantities at the left hand side are the probability distributions which quantum mechanics attaches to the outcomes $\{a, b, \ldots, z\}$ of the considered measurements when the system is in the state $|\psi\rangle$. A deterministic hidden variable model is a particular instance of a stochastic one where all probabilities $P_{\lambda}$ can take only the values 0 or 1 .

\footnotetext{
*Work supported in part by Istituto Nazionale di Fisica Nucleare, Sezione di Trieste, Italy.

${ }^{\dagger}$ Electronic address: ghirardi@ts.infn.it

‡Electronic address: marinatto@ts.infn.it
} 
The considered framework is extremely general and the parameters $\lambda$ may be completely or only partially accessible, both practically or in principle, to the experimenter.

In the case in which the measurement processes take place at spacelike separated locations, the following condition demanding that all conceivable probability distributions of measurement processes satisfy the factorization property

$$
P_{\lambda}\left(A_{i}=a, B_{j}=b, \ldots, Z_{k}=z\right)=P_{\lambda}\left(A_{i}=a\right) P_{\lambda}\left(B_{j}=b\right) \ldots P_{\lambda}\left(Z_{k}=z\right) \quad \forall \lambda \in \Lambda,
$$

is a physically natural one which every hidden variable model is requested to satisfy.

This factorizability request is commonly known as Bell's locality condition [7]. We remark that all "nonlocality without inequalities" proofs aim at exhibiting a conflict between the quantum predictions for a specific entangled state and any local completion of quantum mechanics which goes beyond quantum mechanics itself. In fact, in the particular case in which the most complete specification of the state of a physical system is represented by the knowledge of the state vector $|\psi\rangle$ alone, i.e., within ordinary quantum mechanics, the failure of the locality condition of Eq. (2) can be established directly by plugging into it appropriate quantum mechanical observables. Indeed, it is a well-known fact that for any entangled state there exist joint probabilities which do not factorize and, consequently, that ordinary quantum mechanics is inherently a nonlocal theory.

As it has been noted by A. Fine [8] local stochastic hidden variable models are equivalent to local deterministic hidden variable models. Nonetheless, in what follows we prefer working within the stochastic scenario. This choice, which differs from the one usually made in the literature [1, 4, 5, 6], allows us to express the locality request as the factorizability condition on joint probabilities instead of resorting to Einstein locality [2].

To start with, we consider an $n$-partite system described by the state vector $|\psi(1, \ldots, n)\rangle$ belonging to the Hilbert space $\mathcal{H}_{1} \otimes \cdots \otimes \mathcal{H}_{n}$ - where the dimension of $\mathcal{H}_{i}, i=1, \ldots, n$ is greater than or equal to 2 . Let us split arbitrarily the $n$ particles in two subsets, which we will label for convenience as 1 and 2 in what follows, and suppose they involve particles laying into two space-like separated spatial regions. Finally, let us consider the Schmidt decomposition of $|\psi\rangle$ in terms of the orthonormal sets of states $\left\{\left|\alpha_{i}(1)\right\rangle\right\}$ and $\left\{\left|\beta_{i}(2)\right\rangle\right\}$, referring to the first and to the second group of particles respectively:

$$
|\psi(1,2)\rangle=\sum_{i} p_{i}\left|\alpha_{i}(1)\right\rangle \otimes\left|\beta_{i}(2)\right\rangle,
$$

where the positive weights $p_{i}$ satisfy the normalization condition $\sum_{i} p_{i}^{2}=1$. Suppose now that in Eq.(3) at least two different weights appear which we assume for simplicity to be the first two, so that $p_{1} \neq p_{2}$. Actually, this is the only hypothesis which is required by our proof. This means that the nonlocality without inequalities proof we are going to present holds for those non-completely factorizable states whose Schmidt coefficients are not all equal [9]. In terms of the coefficients $p_{1}$ and $p_{2}$, we build up the following two $2 \times 2$ unitary matrices $U$ and $V$ :

$$
U=\frac{1}{\sqrt{p_{1}+p_{2}}}\left[\begin{array}{cc}
\sqrt{p_{2}} & -i \sqrt{p_{1}} \\
-i \sqrt{p_{1}} & \sqrt{p_{2}}
\end{array}\right] V=\frac{1}{\sqrt{p_{1}^{2}+p_{2}^{2}-p_{1} p_{2}}}\left[\begin{array}{cc}
-i\left(p_{2}-p_{1}\right) & \sqrt{p_{1} p_{2}} \\
\sqrt{p_{1} p_{2}} & -i\left(p_{2}-p_{1}\right)
\end{array}\right] .
$$

We define now two orthonormal bases $\left\{\left|x_{+}(1)\right\rangle,\left|x_{-}(1)\right\rangle\right\}$ and $\left\{\left|y_{+}(1)\right\rangle,\left|y_{-}(1)\right\rangle\right\}$ of the two-dimensional linear manifold of the first group of particles spanned by the vectors $\left\{\left|\alpha_{1}(1)\right\rangle,\left|\alpha_{2}(1)\right\rangle\right\}$, and two bases $\left\{\left|x_{+}(2)\right\rangle,\left|x_{-}(2)\right\rangle\right\}$ and $\left\{\left|y_{+}(2)\right\rangle,\left|y_{-}(2)\right\rangle\right\}$ of the two-dimensional linear manifold of the second group of particles spanned by the vectors $\left\{\left|\beta_{1}(2)\right\rangle,\left|\beta_{2}(2)\right\rangle\right\}$, according to:

$$
\begin{array}{ll}
{\left[\begin{array}{l}
\left|x_{+}(1)\right\rangle \\
\left|x_{-}(1)\right\rangle
\end{array}\right]=U\left[\begin{array}{l}
\left|\alpha_{1}(1)\right\rangle \\
\left|\alpha_{2}(1)\right\rangle
\end{array}\right]} & {\left[\begin{array}{l}
\left|y_{+}(1)\right\rangle \\
\left|y_{-}(1)\right\rangle
\end{array}\right]=V U\left[\begin{array}{l}
\left|\alpha_{1}(1)\right\rangle \\
\left|\alpha_{2}(1)\right\rangle
\end{array}\right]} \\
{\left[\begin{array}{l}
\left|x_{+}(2)\right\rangle \\
\left|x_{-}(2)\right\rangle
\end{array}\right]=U\left[\begin{array}{l}
\left|\beta_{1}(2)\right\rangle \\
\left|\beta_{2}(2)\right\rangle
\end{array}\right]} & {\left[\begin{array}{l}
\left|y_{+}(2)\right\rangle \\
\left|y_{-}(2)\right\rangle
\end{array}\right]=V U\left[\begin{array}{l}
\left|\beta_{1}(2)\right\rangle \\
\left|\beta_{2}(2)\right\rangle
\end{array}\right] .}
\end{array}
$$

From Eqs. (56), one immediately sees that the state $|\psi\rangle$ of Eq. (31) can be expressed in the following three equivalent forms:

$$
\begin{aligned}
|\psi(1,2)\rangle & =i \sqrt{p_{1} p_{2}}\left[\left|x_{+}(1)\right\rangle\left|x_{-}(2)\right\rangle+\left|x_{-}(1)\right\rangle\left|x_{+}(2)\right\rangle\right]+\left(p_{2}-p_{1}\right)\left|x_{-}(1)\right\rangle\left|x_{-}(2)\right\rangle+\sum_{i>2} p_{i}\left|\alpha_{i}(1)\right\rangle\left|\beta_{i}(2)\right\rangle \\
& =i \sqrt{p_{1}^{2}+p_{2}^{2}-p_{1} p_{2}}\left|y_{-}(1)\right\rangle\left|x_{-}(2)\right\rangle+i \sqrt{p_{1} p_{2}}\left|x_{-}(1)\right\rangle\left|x_{+}(2)\right\rangle+\sum_{i>2} p_{i}\left|\alpha_{i}(1)\right\rangle\left|\beta_{i}(2)\right\rangle \\
& =i \sqrt{p_{1} p_{2}}\left[\left|x_{+}(1)\right\rangle\left|x_{-}(2)\right\rangle+i \sqrt{p_{1}^{2}+p_{2}^{2}-p_{1} p_{2}}\left|x_{-}(1)\right\rangle\left|y_{-}(2)\right\rangle+\sum_{i>2} p_{i}\left|\alpha_{i}(1)\right\rangle\left|\beta_{i}(2)\right\rangle .\right.
\end{aligned}
$$


Let us now denote by $X_{1}, Y_{1}, X_{2}$ and $Y_{2}$ the four observables, having as eigenstates associated to the eigenvalues +1 and -1 the previously defined couples of orthogonal vectors $\left\{\left|x_{+}(1)\right\rangle,\left|x_{-}(1)\right\rangle\right\},\left\{\left|y_{+}(1)\right\rangle,\left|y_{-}(1)\right\rangle\right\}$ and $\left\{\left|x_{+}(2)\right\rangle,\left|x_{-}(2)\right\rangle\right\}$, $\left\{\left|y_{+}(2)\right\rangle,\left|y_{-}(2)\right\rangle\right\}$ respectively, while they act as the null operator in the manifolds orthogonal to the bidimensional ones corresponding to the non-zero eigenvalues. According to Eq. (7) and taking into account the orthogonality of the involved states, the quantum joint probabilities concerning the set of observables $X_{1}, Y_{1}, X_{2}$ and $Y_{2}$ satisfy the following relations:

$$
\begin{aligned}
P_{\psi}\left(X_{1}=+1, X_{2}=+1\right) & =0 \\
P_{\psi}\left(Y_{1}=+1, X_{2}=-1\right) & =0 \\
P_{\psi}\left(X_{1}=-1, Y_{2}=+1\right) & =0 \\
P_{\psi}\left(Y_{1}=+1, X_{2}=0\right) & =0 \\
P_{\psi}\left(X_{1}=0, Y_{2}=+1\right) & =0 \\
P_{\psi}\left(Y_{1}=+1, Y_{2}=+1\right) & \neq 0 .
\end{aligned}
$$

Suppose now that a local stochastic hidden variable model reproducing, according to Eq. (1), the quantum predictions for the state $|\psi\rangle$, exists. As a consequence, considering for example Eq. (8), we must have:

$$
\begin{aligned}
P_{\psi}\left(X_{1}=+1, X_{2}=+1\right) & =\int_{\Lambda} d \lambda \rho(\lambda) P_{\lambda}\left(X_{1}=+1, X_{2}=+1\right) \\
& =\int_{\Lambda} d \lambda \rho(\lambda) P_{\lambda}\left(X_{1}=+1\right) P_{\lambda}\left(X_{2}=+1\right)=0
\end{aligned}
$$

where the second equality follows from the request that our model satisfies Bell's locality condition of Eq. (2). Since $\rho(\lambda)$ is a positive distribution, the last integral of Eq. (14) vanishes if and only if the product $P_{\lambda}\left(X_{1}=+1\right) P_{\lambda}\left(X_{2}=+1\right)$ vanishes almost everywhere [10] within $\Lambda$. Analogous reasonings can be applied to Eqs (9.13), leading to:

$$
\begin{array}{r}
P_{\lambda}\left(X_{1}=+1\right) P_{\lambda}\left(X_{2}=+1\right)=0 \\
P_{\lambda}\left(Y_{1}=+1\right) P_{\lambda}\left(X_{2}=-1\right)=0 \\
P_{\lambda}\left(X_{1}=-1\right) P_{\lambda}\left(Y_{2}=+1\right)=0 \\
P_{\lambda}\left(Y_{1}=+1\right) P_{\lambda}\left(X_{2}=0\right)=0 \\
P_{\lambda}\left(X_{1}=0\right) P_{\lambda}\left(Y_{2}=+1\right)=0 \\
P_{\lambda}\left(Y_{1}=+1\right) P_{\lambda}\left(Y_{2}=+1\right)
\end{array}
$$

where the first five equations must hold almost everywhere within $\Lambda$, while the sixth equation has to be satisfied in a subset of $\Lambda$ whose measure according to the distribution $\rho(\lambda)$ is non-zero. It is worth noticing that, in the special case where the parameters $\lambda$ coincide with the state vector $|\psi\rangle$ alone, as happens in ordinary quantum mechanics, the previous equations are immediately seen to be violated. In fact, for the state $|\psi\rangle$ of Eq. (7) both $P_{\lambda=\psi}\left(X_{1}=+1\right)$ and $P_{\lambda=\psi}\left(X_{2}=+1\right)$ are different from zero, thus contradicting Eq. (15).

We prove now, with simple set manipulations, that no conceivable local stochastic hidden variable model exists which is compatible with the previous equations. To this end, let us first of all notice that by summing Eq. (16) and (18) and taking into account that $P_{\lambda}\left(X_{2}=-1\right)+P_{\lambda}\left(X_{2}=+0\right)+P_{\lambda}\left(X_{2}=+1\right)=1$ we have:

$$
P_{\lambda}\left(Y_{1}=+1\right)\left[1-P_{\lambda}\left(X_{2}=+1\right)\right]=0
$$

Similarly, summing Eqs. (17) and (19) we have:

$$
\left[1-P_{\lambda}\left(X_{1}=+1\right)\right] P_{\lambda}\left(Y_{2}=+1\right)=0
$$

Now we define the following subsets $A, B$ and $C$ of $\Lambda$ as:

$$
\begin{aligned}
& A=\left\{\lambda \in \Lambda \mid P_{\lambda}\left(X_{1}=+1\right)=0\right\} \\
& B=\left\{\lambda \in \Lambda \mid P_{\lambda}\left(X_{2}=+1\right)=0\right\} \\
& C=\Lambda-(A \cup B) .
\end{aligned}
$$

Since $\Lambda-(A \cup B)=(\Lambda-A) \cap(\Lambda-B)$, we have that, for all $\lambda$ belonging to $C, P_{\lambda}\left(X_{1}=+1\right) P_{\lambda}\left(X_{2}=+1\right) \neq 0$. If the set $C$ would have a non-zero measure according to the distribution $\rho$, i.e., if $\int_{C} d \lambda \rho(\lambda) \neq 0$, one would violate Eq. (15) and, consequently, Eq. (8). Therefore, in order to fulfill Eq. (15), the set $A \cup B$ must coincide with $\Lambda$ exception made 
for a set of zero measure. We can then argue as follows. If $\lambda$ belongs to $A$ then, by definition, $P_{\lambda}\left(X_{1}=+1\right)=0$, so that, according to Eq. (22) we get $P_{\lambda}\left(Y_{2}=+1\right)=0$. Equivalently, if $\lambda$ belongs to $B$ then $P_{\lambda}\left(X_{2}=+1\right)=0$ and, according to Eq. (21), $P_{\lambda}\left(Y_{1}=+1\right)=0$. Therefore, for any $\lambda \in A \cup B$ either $P_{\lambda}\left(Y_{1}=+1\right)=0$ or $P_{\lambda}\left(Y_{2}=+1\right)=0$, contradicting Eq. (20). We have thus proven that it does not exist a subset of $\Lambda$ of non-zero measure such that Eq. (20) can be satisfied.

This simple argument shows that it is not possible to exhibit any conceivable stochastic hidden variable model, satisfying Bell's locality condition, which can account for the quantum mechanical predictions of almost any $n$-partite quantum entangled state $|\psi\rangle \in \mathcal{H}_{1} \otimes \cdots \otimes \mathcal{H}_{n}$, whenever there exists a splitting of the $n$ particles whose Schmidt decomposition contains at least two different weights.

The experimental test of nonlocality for the considered set of entangled states consists simply in testing the occurrence of the joint measurement outcomes which Eq. (13) shows to be possible. Given the state of Eq. (7), it is straightforward to show that this event happens with a probability:

$$
P_{\psi}\left(Y_{1}=+1, Y_{2}=+1\right)=\frac{p_{1}^{2} p_{2}^{2}\left(p_{1}-p_{2}\right)^{2}}{\left(p_{1}^{2}+p_{2}^{2}-p_{1} p_{2}\right)^{2}}
$$

which does not vanish whenever $p_{1}, p_{2} \neq 0$ and $p_{1} \neq p_{2}$.

The joint probability distribution of Eq. (26) involves measurements of the observables $Y_{1}$ and $Y_{2}$, which are generally multipartite. As well known, from a strict practical point of view, the measurement of such observables might turn out to be not feasible and it would be in any case quite difficult to perform. In order to overcome this problem, we are going to exhibit now a variant of the previous proof which makes reference to repeated application of the Schmidt decomposition of the $n$-partite state in order to identify a non-zero joint probability distribution which conflicts with Bell's locality condition and which involves only single-particle observables.

To accomplish this task, we start by considering a tripartite system and then we show how to generalize the procedure to any number of particles. Consider the Schmidt decomposition of a tripartite state $|\psi(1,2,3)\rangle$ in terms of a set of bipartite orthonormal states of the first and the second particle $\left\{\left|\phi_{k}(1,2)\right\rangle\right\}$, and of a set of orthonormal states of the third particle $\left\{\left|\tau_{k}(3)\right\rangle\right\}$ :

$$
|\psi(1,2,3)\rangle=\sum_{k} q_{k}\left|\phi_{k}(1,2)\right\rangle \otimes\left|\tau_{k}(3)\right\rangle .
$$

where $q_{k} \geq 0$ and $\sum_{k} q_{k}^{2}=1$. Suppose now that, within the orthonormal set $\left\{\left|\phi_{k}(1,2)\right\rangle\right\}$ there exists a state, whose associated $q_{k}$ is different from zero, let us say $\left|\phi_{1}(1,2)\right\rangle$, such that at least two different weights appear in its Schmidt decomposition [11. By resorting to the Schmidt decomposition of such a state and performing the unitary transformations $U$ and $V$ as defined in Eqs. (5.6), the tripartite state $|\psi(1,2,3)\rangle$ exhibits the following form:

$$
\begin{aligned}
|\psi(1,2,3)\rangle= & q_{1}\left(i \sqrt{p_{1} p_{2}}\left[\left|x_{+}(1)\right\rangle\left|x_{-}(2)\right\rangle+\left|x_{-}(1)\right\rangle\left|x_{+}(2)\right\rangle\right]+\left(p_{2}-p_{1}\right)\left|x_{-}(1)\right\rangle\left|x_{-}(2)\right\rangle\right) \otimes\left|\tau_{1}(3)\right\rangle \\
& +q_{1}\left(\sum_{i>2} p_{i}\left|\alpha_{i}(1)\right\rangle\left|\beta_{i}(2)\right\rangle\right) \otimes\left|\tau_{1}(3)\right\rangle+\sum_{k>1} q_{k}\left|\phi_{k}(1,2)\right\rangle \otimes\left|\tau_{k}(3)\right\rangle
\end{aligned}
$$

which can be also rewritten in two other equivalent forms, in analogy with Eq. (7).

Denoting by $T_{3}$ the single-particle observable of the Hilbert space of the third particle having the vectors $\left\{\left|\tau_{k}(3)\right\rangle\right\}$ as its eigenstates associated to a set of eigenvalues $\left\{t_{k}\right\}$ which we may choose so that $t_{1}$ is a non-degenerate eigenvalue, the following quantum probability distributions hold for the state of Eq. (28):

$$
\begin{aligned}
& P_{\psi}\left(X_{1}=+1, X_{2}=+1, T_{3}=t_{1}\right)=0 \\
& P_{\psi}\left(Y_{1}=+1, X_{2}=-1, T_{3}=t_{1}\right)=0 \\
& P_{\psi}\left(X_{1}=-1, Y_{2}=+1, T_{3}=t_{1}\right)=0 \\
& P_{\psi}\left(Y_{1}=+1, X_{2}=0, T_{3}=t_{1}\right)=0 \\
& P_{\psi}\left(X_{1}=0, Y_{2}=+1, T_{3}=t_{1}\right)=0 \\
& P_{\psi}\left(Y_{1}=+1, Y_{2}=+1, T_{3}=t_{1}\right) \neq 0 \text {. }
\end{aligned}
$$

Once again the existence of a local and stochastic hidden variable model reproducing the quantum mechanical prob- 
ability distributions of Eqs. (29 34), implies the following relations:

$$
\begin{array}{r}
P_{\lambda}\left(X_{1}=+1\right) P_{\lambda}\left(X_{2}=+1\right) P_{\lambda}\left(T_{3}=t_{1}\right)=0 \\
P_{\lambda}\left(Y_{1}=+1\right) P_{\lambda}\left(X_{2}=-1\right) P_{\lambda}\left(T_{3}=t_{1}\right)=0 \\
P_{\lambda}\left(X_{1}=-1\right) P_{\lambda}\left(Y_{2}=+1\right) P_{\lambda}\left(T_{3}=t_{1}\right)=0 \\
P_{\lambda}\left(Y_{1}=+1\right) P_{\lambda}\left(X_{2}=0\right) P_{\lambda}\left(T_{3}=t_{1}\right)=0 \\
P_{\lambda}\left(X_{1}=0\right) P_{\lambda}\left(Y_{2}=+1\right) P_{\lambda}\left(T_{3}=t_{1}\right)=0 \\
P_{\lambda}\left(Y_{1}=+1\right) P_{\lambda}\left(Y_{2}=+1\right) P_{\lambda}\left(T_{3}=t_{1}\right) \neq 0 .
\end{array}
$$

As before, the first five equations must be satisfied almost everywhere within $\Lambda$, while the last one must be satisfied in a set of non-zero measure with respect to the distribution $\rho(\lambda)$. Let us now show that a local stochastic hidden variable model satisfying Eqs. (35 40) cannot exist. The procedure we are going to follow consists in splitting the set of hidden variables $\Lambda$ into two complementary and disjoint subsets $\Omega_{1}$ and $\Omega_{2}$, referring to the possible values of the probability distribution for the outcome $t_{1}$ of the observable $T_{3}$. They are defined as $\Omega_{1}=\left\{\lambda \in \Lambda \mid P_{\lambda}\left(T_{3}=t_{1}\right)=0\right\}$ and $\Omega_{2}=\left\{\lambda \in \Lambda \mid P_{\lambda}\left(T_{3}=t_{1}\right) \neq 0\right\}$. Given any value of the hidden variable $\lambda$, two possible cases can occur: either $\lambda \in \Omega_{1}$ or $\lambda \in \Omega_{2}$. If $\lambda \in \Omega_{1}$, the left hand side of Eq. (40) vanishes and the equation cannot be satisfied. If $\lambda$ belongs to $\Omega_{2}$ where $P_{\lambda}\left(T_{3}=t_{1}\right) \neq 0$, the equations from (35) to (40) reduce to Eqs (1520) respectively. This being the case, we can apply the previous arguments to conclude that no local stochastic hidden variable model exists which can reproduce the quantum probabilities for all tripartite states, whose Schmidt decomposition involves at least one bipartite state having at least two different weights in its decomposition. The argument can be generalized in a straightforward way to any number of particles. Note that the $n$-particle entangled states for which our proof holds are those for which, by considering all conceivable Schmidt decompositions in terms of bipartite states and $(n-2)$ single-particle states, at least one entangled bipartite state involving at least two different weights in its Schmidt decomposition appears. Such a set contains almost all entangled states of $n$-particles. Once again, our approach uniquely determines the set of outcomes of joint measurements whose occurrence constitutes the experimental proof of the nonlocality of every conceivable hidden variable completion of quantum mechanics. However, contrary to the first method we have presented, it could be easily implemented in practice since it involves only simple single-particle measurements.

[1] L. Hardy, Phys. Rev. Lett. 71, 1665 (1993).

[2] A. Einstein, N. Rosen and B. Podolsky, Phys. Rev. 47, 777 (1935).

[3] N.D. Mermin, Ann. N.Y. Acad. Sci. 755, 616 (1995).

[4] D.M. Greenberger, M. Horne, and A. Zeilinger in Bell's Theorem, Quantum Theory, and Conceptions of the Universe, M. Kafatos ed., Kluwer, Dordrecht (1989).

[5] Sheldon Goldstein, Phys. Rev. Lett. 72, 1951 (1994).

[6] L. Hardy, in New Developments on Fundamental Problems in Quantum Physics, edited by M. Ferrero and A. van der Merwe, Kluwer, Dordrecht, 163 (1997); D. Boschi, S. Branca, F. De Martini, and L. Hardy, Phys. Rev. Lett. 79, 2755 (1997); A. Cabello, Phys. Rev. Lett. 86, 1911 (2001).

[7] J.S. Bell, Bertlemann's socks and the nature of reality in John S. Bell on The Foundations of Quantum Mechanics, M. Bell, K. Gottfried \& M. Veltman eds., World Scientific (2001).

[8] A. Fine, Phys. Rev. Lett. 48, 291 (1982).

[9] Accordingly, and curiously enough, a proof of the kind we are considering does not apply, for instance, to the maximally entangled states, the ones which exhibit the maximum amount of nonlocal correlations.

[10] By the expression "almost everywhere" it is meant that the argument of the integral may be different from zero at most within a non-empty set $\Gamma$ such that $\int_{\Gamma} d \lambda \rho(\lambda)=0$.

[11] Actually the tripartite states for which our proof holds, are the ones whose Schmidt decomposition, performed by grouping the particles in every possible manner, contains at least one bipartite entangled state for an arbitrary pair of particles which satisfies the above request. 